Filippo Cademartiri

Nico R. Mollet

Giuseppe Runza

Nico Bruining

Ronald Hamers

Pamela Somers

Michiel Knaapen

Stefan Verheye

Massimo Midiri

Gabriel P. Krestin

Pim J. de Feyter

\section{Influence of intracoronary attenuation on coronary plaque measurements using multislice computed tomography: observations in an ex vivo model of coronary computed tomography angiography}

Received: 25 August 2004

Revised: 26 January 2005

Accepted: 1 February 2005

Published online: 5 March 2005

(C) Springer-Verlag 2005

F. Cademartiri $(\bowtie) \cdot$ N. R. Mollet

G. P. Krestin - P. J. de Feyter

Department of Radiology,

Erasmus Medical Center,

Dr. Molenwaterplein, 40,

3015GD Rotterdam, The Netherlands

e-mail: filippocademartiri@hotmail.com

Tel.: +31-10-4634127

Fax: +31-10-4634033

N. R. Mollet · N. Bruining •

R. Hamers · P. J. de Feyter

Department of Cardiology,

Erasmus Medical Center,

Dr. Molenwaterplein, 40,

3015GD Rotterdam, The Netherlands

G. Runza $\cdot$ M. Midiri

Department of Radiology, DIBIMEL,

University of Palermo,

Palermo, Italy

P. Somers · M. Knaapen

Department of Pathology,

Middelheim Hospital,

Antwerp, Belgium

S. Verheye

Department of Cardiology,

Middelheim Hospital,

Antwerp, Belgium

\begin{abstract}
Assessment of attenuation (measured in Hounsfield units, HU) of human coronary plaques was performed using multislice computed tomography (MSCT) in an ex vivo model. In three ex vivo specimens of left coronary arteries in oil, MSCT was performed after intracoronary injection of four solutions of contrast material (400 $\mathrm{mgI} / \mathrm{ml}$ iomeprol). The four solutions were diluted as follows: $1 / \infty, 1 / 200,1 / 80$, and $1 / 20$. All scans were performed with the following parameters: slices/collimation $16 / 0.75 \mathrm{~mm}$, rotation time $375 \mathrm{~ms}$. Each specimen was scored for the presence of atherosclerotic plaques. In each plaque the attenuation was measured in four regions of interest for lumen, plaque (non-calcified thickening of the vessel wall), calcium, and surrounding (oil surrounding the vessel). The results were compared with a one-way analysis of variance test and were correlated with Pearson's test. There were no significant differences in the attenuation of calcium and oil in the four solutions. The mean attenuation in the four solutions for lumen $(35 \pm 10,91 \pm 7$, $246 \pm 18,511 \pm 89 \mathrm{HU}$ ) and plaque
\end{abstract}

$(22 \pm 22,50 \pm 26,107 \pm 36,152 \pm 67 \mathrm{HU})$ was significantly different between each decreasing dilution $(p<0.001)$. The mean attenuation of lumen and plaque of coronary plaques showed high correlation, while the values were significantly different $(r=0.73$; $p<0.001)$. Intracoronary attenuation modifies significantly the attenuation of plaques assessed with MSCT.

Keywords Multislice computed tomography $\cdot$ Coronary angiography Ex vivo experiment - Vascular attenuation $\cdot$ Coronary plaque attenuation

\section{Introduction}

Several studies reported that atherosclerotic plaque composition and morphology are important predictors of plaque stability and clinical behaviour when compared with the degree of vessel stenosis [1-3].
Quantitative and qualitative information (e.g. lumen diameter, wall thickness, and morphology) can be obtained using intracoronary ultrasound (ICUS), which has been approved by Food and Drug Administration as the standard for the in vivo evaluation of coronary plaques [4-6]. This 
technique cannot be used for routine evaluation of plaque characteristics because of its invasiveness and cost.

Several non-invasive techniques have been tested for the visualisation and characterisation of coronary plaques. Preliminary reports using magnetic resonance imaging have reported the ability to detect and characterise the lipid pool in atherosclerotic plaques at the level of the carotid arteries [7], and in the coronary arteries [8-10].

Multislice computed tomography (MSCT) coronary angiography has showed good potential in the detection, quantification, and characterisation of coronary artery plaques [11-16]. Nevertheless, several aspects concerning the methodology for the assessment of coronary plaques with MSCT coronary angiography remain unexplored.

With this study we address the issue of the variability of coronary plaque attenuation as measured with MSCT in an ex vivo model with varying intracoronary attenuation.

\section{Materials and methods}

\section{Specimens}

Three left coronary arteries were dissected during autopsy. The dissected arteries were sampled $1 \mathrm{~cm}$ proximal to the bifurcation of the LCx and covered a length of $4 \mathrm{~cm}$. For orientation the anterior side of the arteries was inked in black and the left side of the artery in green. Two patients (both males, with ages of 78 and 63 years) died of noncardiovascular diseases. One patient (female, with an age of 73 years) died of ischaemic heart disease. The study was approved by the Medical Committee of the Middelheim Hospital.

The specimens were prepared and scanned separately. Each specimen was prepared with two sheaths. The sheaths were introduced and fixed at the proximal end (in the left main coronary artery) and at the distal end (in the anterior descending artery) of the specimens. The left circumflex was, previously, closed at its end with a wire.

\section{Contrast material}

Four saline solutions with decreasing dilution of contrast material (400 $\mathrm{mgI} / \mathrm{ml}$ iomeprol, Bracco, Italy) were used: $1 / \infty, 1 / 200,1 / 80$, and $1 / 20$. The attenuation values (Hounsfield units, HU) of the four solutions obtained in a 10 -ml syringe after dilution were $3.2 \pm 4.5 \mathrm{HU}(1 / \infty$; defined as saline; no contrast material was diluted), $144.7 \pm$ $8.6 \mathrm{HU}(1 / 200$; defined as low), $298.4 \pm 3.4 \mathrm{HU}(1 / 80$; defined as medium), and 588.0 $\pm 6.0 \mathrm{HU}(1 / 20$; defined as high).

\section{Experimental settings}

The experimental settings included a box that was filled with vegetable oil. Prior to positioning the specimen in the oil, saline was instilled through the sheaths to wash out as much as possible. The specimens were put into oil as a means of simulating the epicardial fat. Once the specimen had sunk into the oil, the solution was injected through the sheath using a $10-\mathrm{ml}$ syringe from the sheath positioned at the proximal end of the specimen. The injection was finished when the solution was observed leaking out of the specimen. The leaking solution was removed from the specimen using an empty syringe. The specimen was not mobilised between the scans.

\section{Scan parameters}

A MSCT scan (Sensation 16, Siemens, Germany) was performed after intracoronary injection with increasing concentration of contrast material. After each scan the coronary arteries were washed out with saline before the injection of the following solution and the leaking solution was removed from the specimen using an empty syringe.

All scans were performed with the following parameters: slices/collimation $16 / 0.75 \mathrm{~mm}$, rotation time $375 \mathrm{~ms}$, feed/ rotation $3.0 \mathrm{~mm}$ (pitch 0.25 ), $120 \mathrm{kV}, 400 \mathrm{mAs}$, effective slice thickness $1 \mathrm{~mm}$, reconstruction increment $0.5 \mathrm{~mm}$, field of view (FOV) $100 \mathrm{~mm}$, convolution filter medium

Table 1 Summary of the attenuation values measured in the different solutions in each slice

\begin{tabular}{lcccccc}
\hline & Overall $(\mathrm{HU})$ & Slope & \multicolumn{2}{l}{ Solutions (HU) } & & Medium \\
\cline { 5 - 7 } & & & Saline & Low & High \\
\hline Lumen & $223.0 \pm 186.5^{*}$ & $153.4 \pm 30.7^{*}$ & $35.2 \pm 9.7^{* *}$ & $90.6 \pm 6.7^{* *}$ & $246.2 \pm 17.8^{* *}$ & $511.0 \pm 88.7^{* *}$ \\
Plaque & $86.1 \pm 71.5^{*}$ & $45.7 \pm 25.7^{*}$ & $21.9 \pm 22.4^{* *}$ & $49.9 \pm 26.3^{* *}$ & $106.7 \pm 35.6^{* *}$ & $152.0 \pm 67.2^{* *}$ \\
Calcium & $640.7 \pm 533.7^{*}$ & $8.0 \pm 31.5^{*}$ & $354.1 \pm 152.2$ & $343.6 \pm 167.7$ & $356.6 \pm 150.0$ & $351.3 \pm 164.3$ \\
Surrounding & $-123.9 \pm 11.1^{*}$ & $0.9 \pm 6.2^{*}$ & $-129.6 \pm 6.9$ & $-127 \pm 12.2$ & $-122 \pm 6.6$ & $-115 \pm 7.1$ \\
\hline
\end{tabular}

$H U$ Hounsfield units

$* p<0.05$

$* * p<0.01$ 
Fig. 1 Mean attenuation of the four structures in the four solutions. The increasing attenuation in the lumen affects mainly the attenuation of the plaque. The calcium and the surrounding are, in contrast, almost not affected.

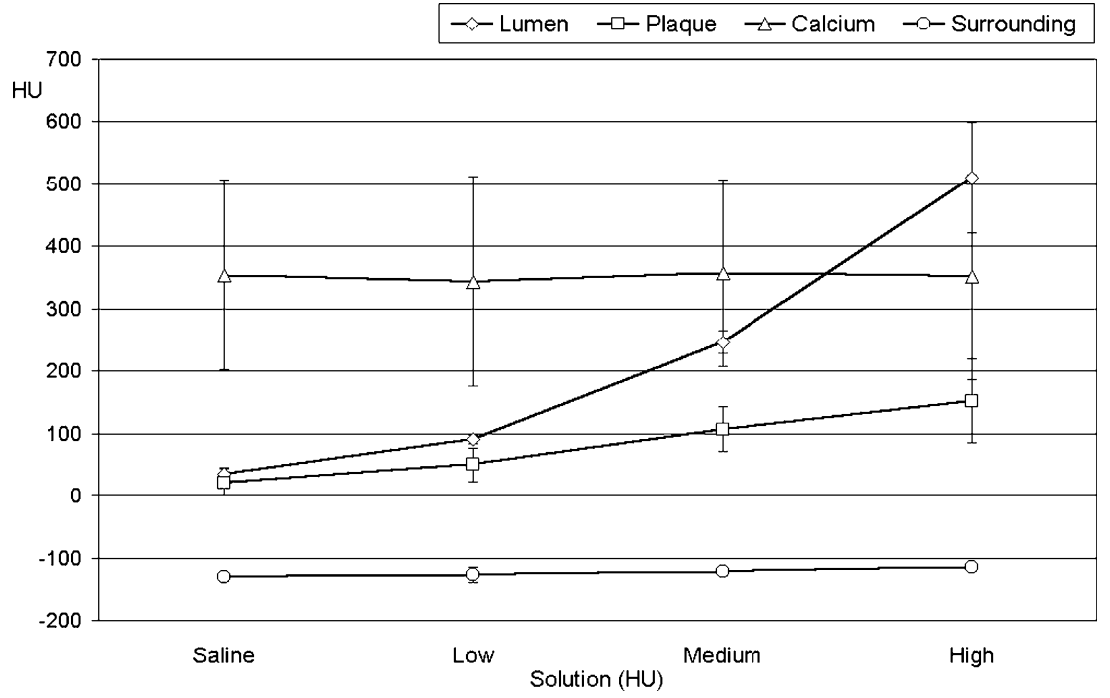

smooth (B30f). The scan geometry was based on a retrospective ECG gated protocol (the same used for in vivo MSCT coronary angiography). This protocol is based on a low pitch that allows a retrospective reconstruction of multiple phases within the cardiac cycle. In this case, a demo ECG was switched on and the scan was performed as if the heart rate was 71 beats per minute. The reconstruction algorithm uses $180^{\circ}$ of rotation, bringing the effective temporal resolution down to $187 \mathrm{~ms}$.

\section{Data preparation}

In order to analyse the specimen with the same settings an experienced operator loaded the datasets into a dedicated workstation (Leonardo, Siemens, Germany) and performed a stack of orthogonal views in each solution for all the specimens with the following parameters: slice thickness $1 \mathrm{~mm}$, increment $0.5 \mathrm{~mm}$, FOV $50 \mathrm{~mm}$.

\section{Data collection}

One observer performed all the measurements. Each specimen was evaluated for the presence of coronary athero-

Table 2 Correlation between the clustered attenuation values in each structure

\begin{tabular}{|c|c|c|c|c|c|}
\hline $\begin{array}{l}\text { Lumen } \\
\text { vs } \\
\text { plaque }\end{array}$ & $\begin{array}{l}\text { Lumen } \\
\text { vs } \\
\text { calcium }\end{array}$ & $\begin{array}{l}\text { Lumen vs } \\
\text { surrounding }\end{array}$ & $\begin{array}{l}\text { Plaque } \\
\text { vs } \\
\text { calcium }\end{array}$ & $\begin{array}{l}\text { Plaque vs } \\
\text { surrounding }\end{array}$ & $\begin{array}{l}\mathrm{m} \text { vs } \\
\text { nding }\end{array}$ \\
\hline $.733^{*}$ & 0.063 & $0.106^{*}$ & $0.178^{* *}$ & $0.166^{* *}$ & 0.060 \\
\hline
\end{tabular}

$* p<0.05$

$* * p<0.01$ sclerotic plaques. A coronary atherosclerotic plaque was defined as a thickening of the coronary wall clearly distinguished from the surrounding hypo-attenuating oil and from the lumen after injection of the solution with the lowest dilution of contrast material (e.g. high). Plaques where targeted regardless of their size.

The operator loaded the four datasets for each dilution of the same specimen onto a workstation screen divided $2 \times 2$, scrolling the datasets in parallel with standard window settings (window width $700 \mathrm{HU}$; window centre $140 \mathrm{HU}$ ). Once a plaque had been detected in the four solutions, the operator drew four regions of interest (ROIs) for contrast material in the lumen of the vessel (defined as lumen), the soft tissue of the coronary plaque (defined as plaque), the calcification within the coronary artery wall (defined as calcium), and the oil immediately surrounding the plaque (defined as surrounding). These four ROIs were defined as structures. The first drawing of the ROI was performed in the dataset with the lowest dilution of contrast material because the lumen was easier to identify. The ROIs were drawn as large as possible but avoiding the borders of each structure in order to limit the effect of interpolation and partial volume on the measurement. Once the four ROIs of the structures had been drawn the operator could copy and paste the ROIs into the other stacks of images corresponding to different solutions. Therefore, the position of the ROIs was mirrored in each orthogonal slice with a plaque for each solution. The mean attenuation in each ROI was collected.

\section{Statistical analysis}

The values of attenuation are presented as means and standard deviations. Statistical evaluation was performed with dedicated software (SPSS 10.1, SPSS, Chicago, IL, 
Fig. 2 Lumen attenuation versus plaque attenuation. All the attenuation values measured within the lumen and the plaque are plotted. The distribution is clearly linear, demonstrating correlation between the two variables $(r=0.73)$.

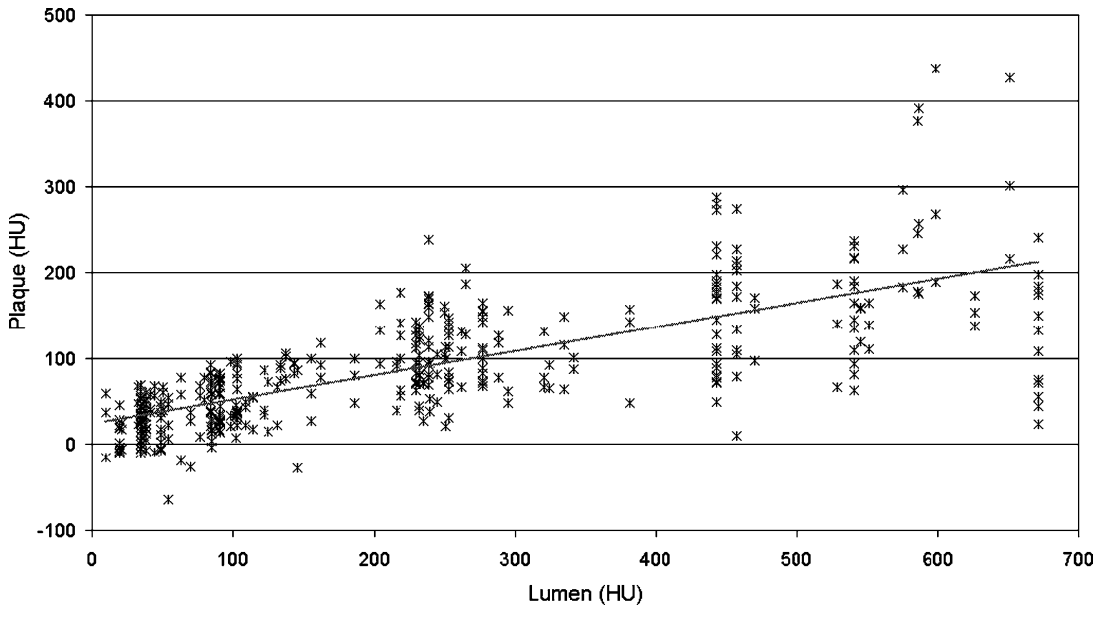

USA). The attenuation measurements in each solution within the structure and clustered per structure were compared with a one-way analysis of variance test and were correlated with Pearson's test. For each structure (e.g. lumen, plaque, calcium, and surrounding), the attenuation values obtained in the four solutions were plotted in order to obtain a slope. The mean slope of each structure was tested for significant differences. For all comparisons tested, $p<0.05$ was considered significant.

\section{Results}

Overall 109 levels (1-mm apart from each other) containing plaque were measured in the three coronary specimens. At each level four solutions were available (436 slices) and in each slice four ROIs were sampled $(1,744$ samples). The results are summarised in Table 1.

The mean attenuation obtained for all samples was $223 \pm$ $187 \mathrm{HU}$ for the lumen, $86 \pm 71 \mathrm{HU}$ for the plaque, $641 \pm$ $534 \mathrm{HU}$ for the calcium, and $-124 \pm 11 \mathrm{HU}$ for the surrounding. The values were all significantly different $(p<0.05)$.

The mean slopes obtained for all the four structures were: $153 \pm 31$ for the lumen, $46 \pm 26$ for the plaque, $8 \pm 31$ for the calcium, and $1 \pm 6$ for the surrounding. Values were all significantly different between the structures $(p<0.05)$.

The mean attenuations in the four solutions for the four structures are displayed in Table 1 and Fig. 1. The attenuation values of the lumen and of the plaques were significantly different in each structure $(p<0.01)$. The attenuation values of the calcium and of the surrounding were not significantly different in each structure $(p>0.05)$.

After clustering the paired attenuation values obtained for the four structures in all the four solutions the correlation between the structures was good $(r=0.733)$ only between the attenuation values of the lumen and the plaque (Table 2, Fig. 2).

\section{Discussion}

Several studies reported the ability of MSCT to visualise coronary atherosclerotic plaque $[12,14,15,17-20]$.

An in vivo study by Kopp et al. [11] compared MSCT and ICUS in the characterisation of coronary plaques. The plaques were divided for the attenuation values into soft ( $6 \pm 28$ and $-5 \pm 25 \mathrm{HU}$; two plaques), intermediate ( $83 \pm 17$ and $51 \pm 19 \mathrm{HU}$; two plaques) and calcified (489 \pm 372 and $423 \pm 111 \mathrm{HU}$; two plaques). The MSCT criteria based on attenuation values showed excellent correspondence with the ICUS criteria.

In an in vivo study, Schroeder et al. [12] analysed the composition of 34 plaques by comparing MSCT and ICUS. They found that plaque echogenicity as determined by ICUS corresponds well to plaque attenuation as measured by MSCT.

Leber et al. [16] in an in vivo study showed that lesion echogenicity correlates well with MSCT attenuation measurements in coronary plaques. The attenuation values for hypoechoic, hyperechoic, and calcified plaques were 49 , 91, and $391 \mathrm{HU}$, respectively. The MSCT attenuation values reflect the predominant plaque composition (e.g. a plaque with a large lipid core with a low echogenicity on ICUS might be identified on the basis of a low attenuation value).

Becker et al. [13] investigated 11 human cadaver heart specimens and compared the atherosclerotic lesions detected by MSCT $(n=40)$ with the histopathological macroscopic characterisation according to American Heart Association criteria. They concluded that MSCT is a promising tool for the characterisation of atherosclerotic coronary lesions.

From the literature cited previously, it appears that MSCT can provide data and characterise coronary artery plaques, on the basis of the attenuation values.

In a phantom study, Schroeder et al. [17] measured the attenuation of two plaques made of rubber material after 
injection within a tube (simulating a coronary artery) of contrast material of three decreasing concentrations: 1:30 (336 HU), 1:40 (280 HU), and 1:50 (258 HU). The increased attenuation of the contrast material determined an increase in the measured attenuation of the plaque. Because of this observation we studied the influence of intravascular attenuation on the assessment of coronary plaques with MSCT.

In our experimental study we showed that lumen attenuation measured by MSCT significantly affects the measured plaque attenuation (Fig. 3). The higher the lumen attenuation, the higher the plaque attenuation. Calcium attenuation and surrounding fat attenuation are, in contrast, not significantly affected. On the basis of this finding, it is difficult to identify absolute ranges of attenuation that relate to the specific plaque characteristics.

Several factors and parameters affect the accuracy of the attenuation measurement. Partial volume and interpolation modify the profiles of attenuation in the range of the soft tissues. This is particularly important when there are calcifications in the plaque and contrast material in the lumen in contiguity with the ROI; therefore, absolute plaque attenuation values determined in the various studies are not comparable.

Our observation has a potential impact on the approach to plaque measurements with MSCT. In fact, since the attenuation within the lumen affects the attenuation within the plaque, further studies should probably focus on the relationship between these two variables. On the basis of our results the relationship appears to be close to linear. Thus, a coefficient could be introduced when plaque measurements are performed in order to compare the different studies.

One of the most important features of the vulnerable plaque is the lipid core. The usual location of the lipid core within the atherosclerotic plaque is just below the thin cap. This means that it is also very close to the attenuation of the lumen as displayed in MSCT. In this area the attenuation measurements will be affected the most by intravascular attenuation.

There are several limitations in our study. The first one is due to the lack of motion in the specimens. The artefacts derived from the heartbeat were not present and/or reproduced. The second limitation is related to the low number of specimens. Nevertheless, the high sampling rate using cross-sectional views of the vessels balanced this. The third limitation is that an excised coronary specimen does not necessarily represent the reality of a coronary vessel. The third limitation is inherent to the lack of a histopathological correlation. Yet, the aim of our study was not to correlate histopathology with MSCT, but to assess the influence of intracoronary attenuation on overall plaque attenuation measurements. The fourth limitation is that we cannot assume that what was described in our study could be directly transferred to different multislice techniques. The fifth limitation is that we did not investigate the influence of convolution kernels on plaque attenuation. Finally, a moving coronary phantom would provide more realistic information regarding plaque attenuation.

In addition it should be taken into account that the introduction of 64-slice CT systems will probably require an additional optimisation of this methodology.

In conclusion, intravascular attenuation modifies significantly the attenuation of the coronary atherosclerotic plaques assessed with MSCT. Therefore, the characterisation of the plaque on the basis of absolute attenuation values should be reported with caution. When plaque attenuation is measured, intraluminal attenuation should also be reported. Probably, a calibration factor will be introduced in the future to address this issue.
Fig. 3 Example of plaque in the four solutions. The scheme in the upper left corner shows the configuration of the plaque in an orthogonal cut performed with multislice computed tomography. The four solutions producing a progressive increase in lumen attenuation are displayed from left to right in the upper part of the figure. Below the relevant attenuations are displayed for every structure.

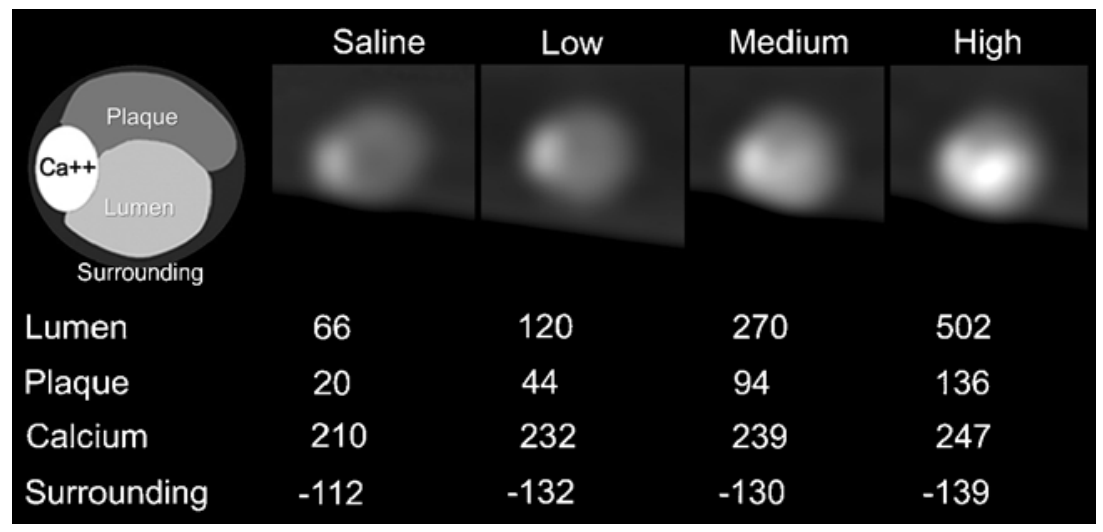




\section{References}

1. Fuster V, Badimon L, Badimon JJ, Chesebro JH (1992) The pathogenesis of coronary artery disease and the acute coronary syndromes (1). N Engl J Med 326:242-250

2. Fuster V, Badimon L, Badimon JJ, Chesebro JH (1992) The pathogenesis of coronary artery disease and the acute coronary syndromes (2). N Engl J Med 326:310-318

3. Virmani R, Kolodgie FD, Burke AP, Farb A, Schwartz SM (2000) Lessons from sudden coronary death: a comprehensive morphological classification scheme for atherosclerotic lesions. Arterioscler Thromb Vasc Biol 20:12621275

4. Stone GW, Hodgson JM, St Goar FG, Frey A, Mudra H, Sheehan H, Linnemeier TJ (1997) Improved procedural results of coronary angioplasty with intravascular ultrasound-guided balloon sizing: the CLOUT pilot trial. Clinical Outcomes with Ultrasound Trial (CLOUT) investigators. Circulation 95:2044-2052

5. Schroeder S, Baumbach A, Haase KK, Oberhoff M, Marholdt H, Herdeg C, Athanasiadis A, Karsch KR (1999) Reduction of restenosis by vessel size adapted percutaneous transluminal coronary angioplasty using intravascular ultrasound. Am J Cardiol 83:875-879

6. Bruining N, Sabate M, de Feyter PJ, Kay IP, Ligthart J, Disco C, Kutryk MJ, Roelandt JR, Serruys PW (1999) Quantitative measurements of in-stent restenosis: a comparison between quantitative coronary ultrasound and quantitative coronary angiography. Catheter Cardiovasc Interv 48:133-142

7. Shinnar M, Fallon JT, Wehrli S, Levin M, Dalmacy D, Fayad ZA, Badimon JJ, Harrington M, Harrington E, Fuster V (1999) The diagnostic accuracy of ex vivo MRI for human atherosclerotic plaque characterization. Arterioscler Thromb Vasc Biol 19:2756-2761
8. Stuber M, Botnar RM, Danias PG, Sodickson DK, Kissinger KV, Van Cauteren M, De Becker J, Manning WJ (1999) Double-oblique free-breathing high resolution three-dimensional coronary magnetic resonance angiography. J Am Coll Cardiol 34:524-531

9. Bunce NH, Pennell DJ (1999) Coronary MRA - a clinical experience in Europe. J Magn Reson Imaging 10:721-727

10. Worthley SG, Helft G, Fuster V, Zaman AG, Fayad ZA, Fallon JT, Badimon JJ (2000) Serial in vivo MRI documents arterial remodeling in experimental atherosclerosis. Circulation 101:586589

11. Kopp AF, Schroeder S, Baumbach A, Kuettner A, Georg C, Ohnesorge B, Heuschmid M, Kuzo R, Claussen CD (2001) Non-invasive characterisation of coronary lesion morphology and composition by multislice CT: first results in comparison with intracoronary ultrasound. Eur Radiol 11:1607-1611

12. Schroeder S, Kopp AF, Baumbach A, Meisner C, Kuettner A, Georg C, Ohnesorge B, Herdeg C, Claussen CD, Karsch KR (2001) Noninvasive detection and evaluation of atherosclerotic coronary plaques with multislice computed tomography. J Am Coll Cardiol 37:1430-1435

13. Becker CR, Nikolaou K, Muders M, Babaryka G, Crispin A, Schoepf UJ, Loehrs U, Reiser MF (2003) Ex vivo coronary atherosclerotic plaque characterization with multi-detector-row CT. Eur Radiol 13:2094-2098

14. Nikolaou K, Sagmeister S, Knez A, Klotz E, Wintersperger BJ, Becker CR, Reiser MF (2003) Multidetector-row computed tomography of the coronary arteries: predictive value and quantitative assessment of non-calcified vesselwall changes. Eur Radiol 13:25052512

15. Achenbach S, Moselewski F, Ropers D, Ferencik M, Hoffmann U, MacNeill B, Pohle K, Baum U, Anders K, Jang IK, Daniel WG, Brady TJ (2004) Detection of calcified and noncalcified coronary atherosclerotic plaque by contrastenhanced, submillimeter multidetector spiral computed tomography: a segment-based comparison with intravascular ultrasound. Circulation 109:14-17
16. Leber AW, Knez A, Becker A, Becker C, von Ziegler F, Nikolaou K, Rist C, Reiser M, White C, Steinbeck G, Boekstegers P (2004) Accuracy of multidetector spiral computed tomography in identifying and differentiating the composition of coronary atherosclerotic plaques: a comparative study with intracoronary ultrasound. J Am Coll Cardiol 43:1241-1247

17. Schroeder S, Flohr T, Kopp AF, Meisner C, Kuettner A, Herdeg C, Baumbach A, Ohnesorge B (2001) Accuracy of density measurements within plaques located in artificial coronary arteries by X-ray multislice CT: results of a phantom study. J Comput Assist Tomogr 25:900-906

18. Leber AW, Knez A, White CW, Becker $\mathrm{A}$, von Ziegler F, Muehling O, Becker C, Reiser M, Steinbeck G, Boekstegers P (2003) Composition of coronary atherosclerotic plaques in patients with acute myocardial infarction and stable angina pectoris determined by contrastenhanced multislice computed tomography. Am J Cardiol 91:714-718

19. Schoenhagen P, Tuzcu EM, Stillman AE, Moliterno DJ, Halliburton SS, Kuzmiak SA, Kasper JM, Magyar WA, Lieber ML, Nissen SE, White RD (2003) Non-invasive assessment of plaque morphology and remodeling in mildly stenotic coronary segments: comparison of 16-slice computed tomography and intravascular ultrasound. Coron Artery Dis 14:459-462

20. Nikolaou K, Becker CR, Muders M, Babaryka G, Scheidler J, Flohr T, Loehrs U, Reiser MF, Fayad ZA (2004) Multidetector-row computed tomography and magnetic resonance imaging of atherosclerotic lesions in human ex vivo coronary arteries. Atherosclerosis 174:243-252 